Dakin, H. D. (1941). J. biol. Chem. 140, 847.

Foreman, F. W. (1914). Biochem. J. 8, 463.

Graff, S., Maculla, E. \& Graff, A. M. (1937). J. biol. Chem. $121,81$.

Gulland, J. M. \& Morris, C. J. O. R. (1934). J. chem. Soc. p. 1644.

Harington, C. R. \& Randall, S. S. (1931). Biochem. J. $25,1917$.

Jones, D. B. \& Moeller, O. (1928). J. biol. Chem. 79, 429.

Kassell, B. \& Brand, E. (1938). J. biol. Chem. 125, 145.

Kossel, A. \& Gross, R. E. (1924). Hoppe-Seyl. Z. 135, 167.

Lucas, C. C. \& Beveridge, J. M. R. (1940). Biochem. J. $34,1356$.

Mueller, J. H. (1923). J. biol. Chem. 56, 157.

Nicolet, B. H. \& Shinn, L. A. (1939). J. Amer. chem. Soc. 61, 1615 .
Nicolet, B. H. \& Shinn, L. A. (1942). J. biol. Chem. 142, 139.

Ritthausen, H. (1869). J. pralkt. Chem. 108, 445.

Robison, R. (1922). Biochem. J. 16, 134.

Shinohara, K. (1936). J. biol. Chem. 112, 683.

Sullivan, M. X. \& McCarthy, T. E. (1940). J. biol. Chem. 188, Proc. xxxiv.

Trotman, S. R. \& Bell, H. S. (1926). J. Soc. chem. Ind., Lond., 45, 10T.

Van Slyke, D. D., Hiller, A., MacFadyen, D. A., Hastings, A. B. \& Klemperer, F. W. (1940). J. biol. Chem. $138,287$.

Vickery, H. B. (1940). J. biol. Chem. 132, 325.

— \& Leavenworth, C. S. (1928a). J. biol. Chem. 76, 707.

- (1928b). J. biol. Chem. 79, 377. (1929). J. biol. Chem. 83, 523.

\& White, A. (1933). J. biol. Chem. 99, 701.

\title{
The Analysis of Hair Keratin
}

\section{ISOLATION OF PROLINE FROM HUMAN HAIR}

\author{
By J. M. R. BEVERIDGE AND C. C. LUCAS, Banting and Best Department of Medical Research, \\ University of Toronto, Toronto, Canada
}

\section{(Received 3 January 1944)}

Previous communications from this laboratory have described the attempted quantitative isolation of the amino-acids of human-hair hydrolysates. Tó date, the separations of cystine (Lucas \& Beveridge, 1940), and the dicarboxylic and basic amino-acids (Beveridge \& Lucas, 1944) have been reported. This paper gives details of further treatment of the amino-acids remaining in the hydrolysate, which has led to the isolation of proline and has indicated the probable absence of hydroxyproline. No data concerning these amino-acids in human hair have as yet appeared in the literature.

In the preceding papers comment was made upon the importance of the order of separation of the amino-acids. Without going into the experimental details, it may be mentioned that four attempts to isolate proline quantitatively were made, with variations in the reagents used and in the stage of the analysis at which they were applied. Precipitation with ammonium reineckate (Kapf hammer \& Eck, 1927), after removal of the cystine, the dicarboxylic acids and arginine, was quickly and easily carried out, but subsequent removal of the reineckate from the proline fraction and from the filtrate was a laborious and time-consuming procedure. The scheme described below gave excellent results and appeared more suitable for a systematic separation of the amino-acids such as was being attempted.

\section{EXPERIMENTAL}

The filtrate from the phosphotungstic acid precipitation of Jysine (Lucas \& Beveridge, 1940; Beveridge \& Iucas, 1944) was freed from inorganic ions in the usual way. The mother liquors from the purification of lysine, as picrate, were freed from picric acid and returned to the main solution. The resulting solution was therefore a human-hair hydro. lysate from which melanin, cystine, arginine, tyrosine, ammonia, dicarboxylic acids, histidine and lysine had been removed, in that order, and in as quantitative a manner as possible.

The solution was concentrated in vacuo and allowed to stend in the room for some days, during which one large crop and several smaller ones of leucine crystallized and were filtered off. (Found: N, 10.67. Calc.: N, 10.69\%.) These accounted for about $7 \%$ of the hair keratin. No attempt has been made as yet to distinguish between leucine, norleucine and isoleucine in the material isolated.

The materials removed from the hydrolysate up to this point account for $58 \%$ of the original weight of hair keratin, i.e. about one-half of the products of hydrolysis have been removed and identified at this stage. The $N$ accounted for amounts to $68 \%$ (including $7 \%$ lost by adsorption on inorganic procipitates).

The remaining amino-acids were converted to copper salts by repeated evaporation with an excess of copper carbonate. (The commercial preparation of copper carbonate available here contained small amounts of soluble inorganic salts which were leached out with warm water before use.) The resulting copper salts were separated into water-insoluble (fraction A) and water-soluble salts (fraction B) as described by Town (1928), Brazier (1930) and Damodaran (1931).

Fraction A was relatively small, since most of the amino. acids which form water-insoluble copper salts had already been removed. It has not yet been examined.

The aqueous extract (fraction B) was ev́aporated, and salts were granulated and dried with acetone in the usual manner (Town, 1928). By repeated extraction with absolute methanol the copper salts more soluble in this solvent (fraction B.2) were separated from those insoluble 
(fraction B-1). As other workers have found, this crude fraction B-2 would not all redissolve in absolute methanol. The residue obtained by evaporating the methanol solution was re-extracted and this process was repeated four times more until the residue was completely soluble in methanol. The insoluble residues were returned to fraction B-1.

Fraction B-2 was diseolved in water and freed from copper with $\mathrm{H}_{2} \mathrm{~S}$. The resulting copper sulphide was repeatedly extracted with water because of the considerable loss of $\mathrm{N}$ on this precipitate (as much as $6 \mathrm{mg} . \mathrm{N} / \mathrm{g}$. CuS). The solution of free amino-acids was concentrated in vacuo to a creamy semi-solid state; 10 vol. of absolute ethanol were stirred slowly into the paste and the mixture was allowed to stand for some hours. The ethanol-insoluble amino-acids were filtered off. The filtrate was taken to dryness in vacuo and the residue extracted five times with absolute ethanol (5 ml./g. of original methanol-soluble copper salts were used for the first extraction; $3 \mathrm{ml} . / \mathrm{g}$. for the subsequent ones). The ethanol solution was again taken to dryness. This time the residue dissolved at once in absolute ethanol (about $5 \mathrm{ml} . / \mathrm{g}$.); on standing, a very small flocculent precipitate appeared, which was removed and added to the ethanol-insoluble fraction. The solution was made to $250 \mathrm{ml}$. and portions taken for total- and amino-N determinations. The ethanol-soluble material (B-2b) contained about one-half of the $\mathrm{N}$ present in fraction B-2; $76 \%$ of the $\mathrm{N}$ in $\mathrm{B}-2 b$ was in the non-amino form.

The ethanol solution (proline fraction) was concentrated to about $175 \mathrm{ml} ., 5 \%$ water added and a slight excess of a saturated solution of $\mathrm{CdCl}_{2} \cdot 2 \mathrm{H}_{2} \mathrm{O}$ in $95 \%(\mathrm{v} / \mathrm{v})$ ethanol was stirred in slowly. A creamy yellow, gummy precipitate came down at once, and during $15 \mathrm{hr}$. in the refrigerator crystalline materiai was deposited on the walls of the vessel. The supernatant liquid was decanted, concentrated and treated with an equal volume of alcoholic $\mathrm{CdCl}_{2}$ solution. Rosettes of crystals appeared when the solution was left in the refrigerator for several days.

The combined $\mathrm{CdCl}_{2}$ precipitates (washed with $95 \%$ $(\nabla / v)$ ethanol) were dissolved in water and freed from inorganic ions. $81 \%$ of the $N$ in this solution was in the non-amino form. Picric acid equivalent to the latter was added to the solution at the boiling-point. Scratching the vessel walls induced the formation of a heavy yellow crystalline deposit. The ice-cold mixture was filtered and the proline picrate was washed with cold picric acid solution, cold ethanol and ether. After drying in vacuo over $\mathrm{P}_{2} \mathrm{O}_{5}$, the picrate melted at $150-151^{\circ}$ (recorded values (Town, 1928), m.p.148 and 152-154 ${ }^{\circ}$ ). Recrystallization did not change the melting-point materially and $95 \%$ of the crop was recovered. A second crop, slightly less pure; was obtained by concentrating the solution. Fractional crystallization of the second crop from water yielded three crops whose melting-points varied from 151 to $153^{\circ}$. The total yield obtained corresponded to $4.3 \%$ proline in human hair. In one experiment where arginine had not been previously removed, as much as $0.4 \%$ of the original keratin was accounted for as arginine isolated as picrate from the final proline fraction.

No hydroxyproline could be found in the human-hair hydrolysate. In one case ammonium reineckate was added to an amino-acid solution obtained after removal of arginine as monoflavianate (cystine and the dicarboxylic acids had been isolated previously from this particular hydrolysate). From a sample of hydrolysate equivalent to $30 \mathrm{~g}$. of hair keratin, crystalline reinecke salt, deep rose-red in colour, was deposited overnight at $0^{\circ}$. The precipitate was washed and decomposed in the usual way, giving finally a solution containing $149 \mathrm{mg}$. of $\mathrm{N}, 94.6 \%$ of which was in the nonamino form. After removal of proline as picrate, the filtrate was freed from picric acid, and another reineckate precipitation was performed in order to precipitate any hydroxyproline which might have been present. That hydroxyproline is absent from human hair seems to be established by the following evidence. This second reineckate precipitate was freed from the reineckate radical and treated with $\mathrm{CdCl}_{2}$ in ethanol, a reagent which has been used to separate proline from hydroxyproline (Kapfhammer \& Eck 1927). The $N$ content of the resulting precipitate was correct for that of the $\mathrm{CdCl}_{2} \cdot \mathrm{H}_{2} \mathrm{O}$ compound of proline (found: $N, 4.2$; calc.: $N, 4.4 \%$ ), and since this precipitate contained practically all of the non-amino $N$ of the second reineckate precipitate the presence of more than small amounts of hydroxyproline was excluded.

Similar results were obtained with another hydrolysate, which was fractionated by quite different procedures. After removal of cystine as cuprous mercaptide, of arginine and histidine as silver salts and of the dicarboxylic acids as barium salts insoluble in ethanol, the remaining aminoacids were subjected to carbamation. The non-carbamating fraction, which should contain the proline and hydroxyproline, was freed from barium. Proline was isolated by precipitation as reineckate and purification via the picrate as described above. A careful search for hydroxyproline in the mother liquors failed to reveal any evidence for its presence. A similar search in the methanol-soluble copper salts derived from the carbamated fraction also yielded negative results.

\section{DISCUSSION}

The isolation of proline from human-hair hydrolysates (previously freed from cystine and arginine) was most easily performed by utilizing the reineckate procedure of Kapfhammer \& Eck (1927). However, for any systematic separation of the amino-acids, it was less suitable because of the labour involved in removing the excess reagent and the losses of $\mathrm{N}$ by adsorption on the many precipitates. After suitable preliminary treatment of the hydrolysate, the procedure recommended by Town (1936) of using the ethanol-soluble amino-acids from the copper salts soluble in methanol proved much more satisfactory.

\section{SUMMARY}

1. Conditions for the quantitative isolation of proline, suitable for use in a systematic scheme of separation of amino-acids from hydrolyzed hair keratin, have been studied.

2. Maximum yield of proline $(4 \cdot 3 \%)$ was obtained by application of Town's copper salt method, after preliminary removal of cystine, arginine, most of the tyrosine, the dicarboxylic acids, histidine, lysine and some leucine had been effected.

3. No hydroxyproline could be found in human. hair hydrolysates. 
This work was aided in part by a grant from the Banting Research Foundation to the Banting and Best Department of Medical Research. Grateful acknowledgement is made to Miss Margaret Wheatley and Mr William Watson for many careful analyses and technical assistance.

\title{
REFERENCES
}

Beveridge, J. M. R. \& Lucas, C. C. .(1944). Biochem. J. Kapfhammer, J. \& Eok, R. (1927). Hoppe-Seyl. Z.170, 294. 38, 88.

Brazier, M. A. B. (1930). Biochem. J. 24, 1188.

Damodaran, M. (1931). Biochem. J. 25, 190.

Lucas, C. C. \& Beveridge, J. M. R. (1940). Biochem.J.34, 1356.

Town, B. W. (1928). Biochem. J. 22, 1083.

(1936). Biochem. J. 30. 1837.

\section{Biochemical Characterization of the Actions of Chemotherapeutic Agents}

\section{MEASUREMENT OF GROWTH OF STREPTOCOCCAL CULTURES THROUGH THEIR GASEOUS METABOLISM, AND THE EFFECTS OF PANTOTHENATE AND PANTOYLTAURINE UPON THE METABOLISM AND GROWTH}

\author{
By H. MoIlWAIN (Leverhulme Research Fellow), Department of Bacterial Chemistry \\ (Medical Research Council), The University, Sheffield
}

(Received 14 January 1944)

During chemotherapeutical experiments with pantoyltaurine, large doses of haemolytic streptococci diseppeared from rats, while in control experiments the animals succumbed to the infection (McIlwain \& Hawking, 1943). In vitro pantoyltaurine did not kill the bacteria but delayed their growth. It has been argued elsewhere (McIlwain, 1943) that such bacteriostasis may afford an adequate basis for many chemotherapeutic actions without the assumption of additional effects of drugs upon the parasites in vivo, or of drugs upon defence mechanisms of the host. The action of pantoyltaurine thus represents a general problem in chemotherapy, namely, the characterization of the in vitro bacteriostasis associated with in vivo disinfection, and the assessing of its contribution to that process. Pantoyltaurine was prepared with the intention of interfering with the parasite's use of pantothenate. The latter, is present in the host, is essential to growth of the bacterium, and is capable, in sufficient quantity, of preventing the action of pantoyltaurine both in vivo and in vitro. The effects of both pantothenate and pantoyltaurine upon streptococcal growth in vitro, in relation to the general problem, have now been investigated.

A manometric method of measuring streptococcal growth was preferred to direct counting, or to opacity measurement, as the organisms are individually small but grow in tangled chains which precipitate quickly. The method permitted continuous readings in standard apparatus, without periodic sampling. Very similar conditions could be used for examining the effects of the reagents upon

Biochem. 1944, 38 streptococcal metabolism; such effects are described here only in so far as they bear upon the use of the method for meesuring growth. Because diffieulties were encountered in the use of streptococcal respiration for growth measurement in pantothenatelimited solutions, and because the anaerobic glycolysis of the organisms involves a gaseous exchange many times that of their oxygen uptake, anaerobic conditions were adopted for growth. These may be more removed from circumstances in vivo than are aerobic conditions, but the difference is unusually small in the case of haemolytic streptococci, especially with respect to their growth in media containing glucose (cf. Hewitt, 1932). No difference in the concentrations of pantothenate and pantoylteurine necessary for growth and inhibition under the two conditions has been found. The manometric method necessarily measures increase of metabolizing units and not of cell numbers. The two factors are known to increase at rates which are not entirely parallel (Huntingdon \& Winslow, 1937); the same is true of cell mass and cell numbers. Increase in cell numbers approximately equal to increase in glycolysis was found during normal growth of the present organisms, when increases of 50- to 100-fold, progressing through the usual phases of growth, were observed. Further correlation of the manometric measure of growth with the usual bacteriological measure by increase in cell numbers has not been attempted as there is no certainty, except when a few cells only are concerned, that the pathogenicity of a normal culture is related to cell numbers rather than to cell mass 